\title{
On oral calcifications: Tartar and Pulp stones
}

\author{
Anjos, A.J. *, Nolasco, P. ${ }^{* *}$, Aquino Marques, J.M. ${ }^{*}$, Alves de Matos, A.P. ${ }^{* * *}$, Carvalho, P.A. ${ }^{* *}$ \\ * Faculty of Dental Medicine, University of Lisbon, Cidade Universitária, 1649-003 Lisbon, Portugal. \\ ** ICEMS, Instituto Superior Técnico, University of Lisbon, Av. Rovisco Pais, 1049-001 Lisboa, Portugal. \\ *** Cooperativa de Ensino Superior - Egas Moniz, Campus Universitário, Quinta da Granja, 2829- 511 Monte \\ de Caparica, Almada, PORTUGAL
}

\section{Email: anacjacome@gmail.com}

The oral cavity is susceptible to several calcifications such as salivary calculi [1] tartar [2], tonsillar concretions [2] and pulp stones [3]. Although several individual studies have been already carried out, a comprehensive morphological and elemental comparison between them is still missing. Sialoliths are most commonly found in the submandibular glands and are composed of regions rich in $\mathrm{Ca}$ and $\mathrm{P}$ minerals, namely hydroxyapatite, whitlockite and brushite, and regions consisting of organic matter with high-sulphur content [1]. Generally, tartar presents an inorganic structure rich in $\mathrm{Ca}$ and $\mathrm{P}$ minerals, such as brushite, octacalcium phosphate, hydroxyapatite and whitlockite, and an organic matrix, mainly constituted by aerobic bacteria and yeast or just anaerobic bacteria [4]. Tonsillar concretions are essentially constituted by bacteria dispersed in a Ca-rich matrix [4]. Pulp stones present needle-like crystallites closely associated with a collagen matrix [3].

The current study is focused on the ultrastructure and chemical characterization of these calcified structures by scanning electron microscopy (SEM) combined with energy dispersive spectroscopy carried out with a JEOL JSM 7001F instrument with an INCA pentaFetx3 Oxford spectrometer operated at $15 \mathrm{kV}$.

The results showed that pulp stones present a subtle layered internal architecture (Fig.1 (a)). At finer scales the structure of pulp stones consist of collagen-like fibres in a mineralized matrix rich in $\mathrm{Ca}$ and P (Fig. 1 (b)). The tartar showed successive accretions that can be associated with a chronological evolution (see arrows in Fig. 1 (c)). At finer scales these calcified materials presented needle-like crystals rich in $\mathrm{Ca}$ and $\mathrm{P}$ (A) as well as bacteria surrounded by droplets (B) (Fig. 1 (d)). Based on the similarities new correlations between the different types of calcifications will be available. For instance, the mineralization process occurring in tartar can help understand the similar processes occurring in sialoliths and tonsilloliths.

The authors acknowledge financial support of the Portuguese Foundation for Science and Technology through PTDC/SAU-ENB/111941/2009 and PEst-OE/CTM-UI0084/2011 grants. Communication submitted to the Microscopy at the Frontiers of Science 2013 congress in Tarragona, Spain.

[1] Alves de Matos, AP. et al., Microscopy and Microanalysis, 11, 152-153, 2005.

[2] Anjos, A.J. et al., Microscopy and Microanalysis, 19, (suppl 4) 23-24, 2013.

[3] Goga, R. et al., International Endodontic Journal, 41, 457-468, 2008.

[4] Charlier, P. et al., Legal Medicine, 12, 163-171, 2010. 


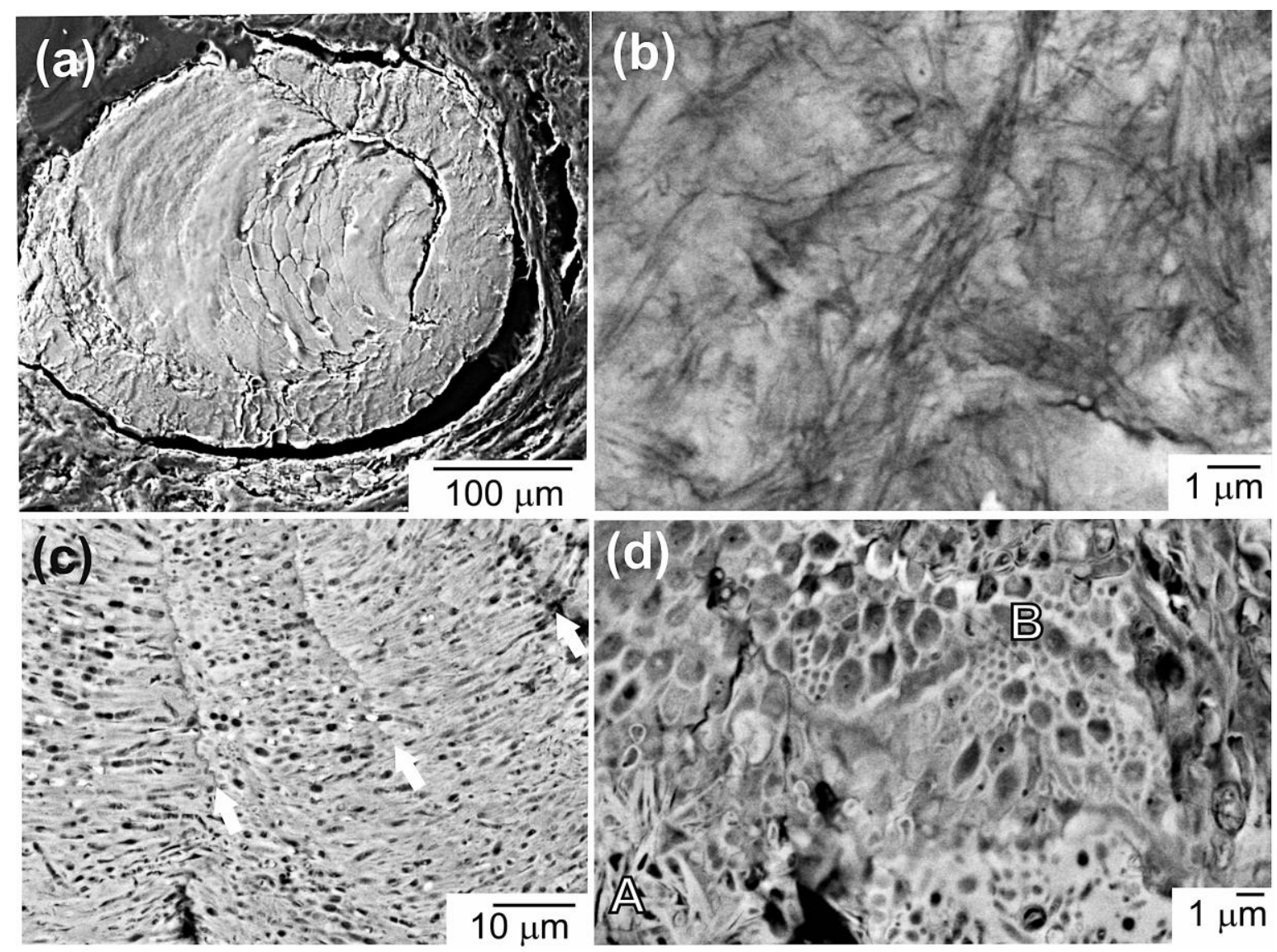

Figure 1. Microstructure of pulp and dental calculi: (a) General morphology of a pulp calculus and (b) the representative local microstructure with collagen-like fibers in a mineralization matrix. (c) General morphology of a dental calculus where the arrows indicate successive deposits and (d) its complex microstructure showing needle-like crystals (A) and bacteria surrounded by droplet structures (B). 